\title{
Computational Modelling in Pyrometallurgy: Part II
}

\author{
M.A. RHAMDHANI ${ }^{1}$ and Q.G. REYNOLDS $\mathbb{1}^{2,3,4}$ \\ 1.-Swinburne University of Technology, John Street, Hawthorn, VIC 3122, Australia. \\ 2.-Mintek, 200 Malibongwe Drive, Randburg, Johannesburg 2194, South Africa. \\ 3.-Stellenbosch University, Private Bag X1, Matieland, Stellenbosch 7602, South Africa. \\ 4.-e-mail: quinnr@mintek.co.za
}

\section{INTRODUCTION}

In Part II of this special topic on Computational Modeling Pyrometallurgy, eleven papers are presented highlighting the wide variation of computational methods applied in the field. As was seen in Part I, pyrometallurgy is a tremendously challenging and complex engineering endeavor involving the handling, processing, and containment of materials in the molten state at very high temperatures. Such extreme environments offer limited opportunities for gathering experimental data, and advanced computational models are valuable additions to the researcher's arsenal of tools for filling inevitable gaps in the existing body of knowledge. When used as virtual prototypes, computational models also afford the unique opportunity to conduct safe, fast, cheap testing of process design changes in order to optimize them before implementation in the real world.

Today, computational modeling finds such wide application across the pyrometallurgy value chain in so many different ways that it is arguably far too broad a topic! The set of papers presented here covers topics from fundamental academic research into solution methods and techniques, through applications and process discovery, to the development and testing of pragmatic solutions to engineering problems. This demonstrates that the digitalization of the pyrometallurgy $R \& D$ pipeline is maturing, and computational modeling using bottom-up approaches based on fundamental physics and chemistry is well positioned to complement and enhance the current explosion in top-down data-centric modeling driving Industry 4.0.

Quinn Reynolds and M Akbar Rhamdhani are guest editors for the topic Computational Modelling in Pyrometallurgy in this issue. The topic is sponsored by the TMS Pyrometallurgy Committee.

(Received July 29, 2021; accepted August 2, 2021; published online August 12, 2021)

\section{PAPERS IN THIS ISSUE}

Multiphase fluid flow is ubiquitous in pyrometallurgy. The large differences in scale between phase interfaces $(\mu \mathrm{m})$ and furnace vessels $(\mathrm{m})$ make it extremely challenging to capture small-scale interfacial flow phenomena accurately inside large-scale engineering models. The development and improvement of algorithms to do this is an active area of research in computational fluid dynamics modeling. In "Toward Multiscale Model Development for Multiphase Flow: Direct Numerical Simulation of Disperse Phases and Multiscale Interfaces in a GasStirred Ladle," authors Q. Li and P.C. Pistorius present an interesting new method for dispersedphase modeling and apply it to the problem of gasstirring in steel ladles.

The application of computational materials science to problems in pyrometallurgical engineering is a rapidly developing field. Such methods permit the study of thermochemical reactions, phase changes, and thermophysical materials properties by directly modeling the quantum mechanical behavior of the constituent atoms themselves; a more fundamental approach is hard to imagine! In "A First-Principles Tool to Discover New Pyrometallurgical Refining Options," M.J. van Setten et al. offer a glimpse into a future in which it is possible to design entirely new pyrometallurgical processes using computational modeling alone.

Purging plugs in ladles need to have a long life for a more stable operation of ladle metallurgy processes. In "Numerical Analysis of the Irreversible Behavior of Corundum Castable Purging Plugs During Service," F. Tan et al. carried out a finite element modeling using a material constitutive model (Drucker-Prager) to investigate failure mechanisms of the plug due to thermochemical load. The study demonstrated a more significant and early shear failure occurs in plugs with rectangular slits than in those with circular slits. The 
results provide some information on the location and mechanism of the failures that can be used to guide the operation practice.

$\mathrm{RH}$ degassing is one of important steps to produce high-grade clean steel. In this process, dissolved gases are removed by circulating steel into a vacuum chamber. In this article, "Numerical Simulation of Gas-Steel-Slag Multiphase Flow in the Vacuum Chamber of the RH Degasser," B. Wang et al. used a particle-free surface-coupled model to investigate multiphase flow in a 210-ton $\mathrm{RH}$ degasser. The gas-steel interpenetration motion, the fluctuation on the free surface, and the behavior of the slag are investigated. The results provide insights useful for industrial practice.

There have been many efforts around the world to effectively inject more reducing gas through blast furnace tuyeres to reduce the overall coke requirement in the reactor. In "Influence of Reducing Gas Injection Methods on Pulverized Coal Combustion in a Medium Oxygen-Enriched Blast Furnace," authors X. Peng et al. attempted to model different scenarios/configuration of the injection of reducing gas and oxygen and their effect on the combustion behavior in the blast furnace (e.g., on the gas velocity distribution, temperature distribution, pulverized coal particle trajectory, and other key phenomena). It was demonstrated that higher volumes of reducing gas can be beneficial, but can cause erosion to the tuyeres. The results presented in the study provide some useful information for the development of a practical approach in reducing gas injection in a blast furnace.

The Peirce-Smith converter design consists of a rotatable horizontal cylinder lined with injection tuyeres through which oxygen-rich gas is injected into crude molten metal, and is ubiquitous across a wide range of industries from copper smelting to platinum-group metal production. Injection of gases into the vessel generally takes place at transonic or supersonic velocities, leading to extremely violent multiphase flow of the slag and metal phases inside. As demonstrated in "Numerical Simulation of Nozzle Height on the Effect of Fluid Flow in a PeirceSmith Converter" by T. Lu et al., computational fluid dynamics modeling can give tremendous insight into the flow and mixing patterns inside such vessels, permitting rapid evaluation of proposed design changes in silico prior to implementation.

Interaction between gas jets and molten materials is a common phenomenon in pyrometallurgical processing, particularly in secondary treatment units such as ladle furnaces and converters. Depending on the momentum of the impinging jet, the resulting deformation of the liquid surface can range from minimal to extreme, affecting the interfacial surface area available for chemical reactions and mass transfer. In "Cavity Formation Induced by a Gas Jet Impinging on a Liquid
Surface Based on a Deformed Mesh Method," X. Zhou et al. have investigated an adaptive meshing method which follows the gas-fluid interface as it deforms, permitting accurate prediction of the surface shape as a function of the jet parameters and fluid properties.

The life of BOF lances is important for the overall process stability and efficient refining in the reactor for steelmaking. Mixed oxygen-powder injection through the lance is sometimes applied in the process. In "A Numerical Study on Particle-Induced Erosion to a Laval Nozzle in Powder-Oxygen Injection of a BOF Lance," M. Li and co-authors modeled an erosion behaviour of mixed gas-powder through a laval nozzle. Various process parameters were considered and evaluated in the study. The erosion was found to be non-uniform with the highest at the nozzle convergent section. The study suggested a particular feeding rate and particle size that can minimize erosion.

As a key raw material for the rapidly developing clean energy sector, silicon is an increasingly important commodity. Its production is a chemically-complex and energy intensive process, the first step of which is usually pyrometallurgical: the reductive smelting of silica to crude silicon in submerged-arc electric furnaces. The interiors of such furnaces are complex and non-uniform in structure, making the prediction of their electrical behavior extremely challenging. "The Effect of Electrode Movements on Electrical Characteristics of a Submerged Arc Furnace" by Y. A. Tesfahunegn et al. applies computational models to the problem, enabling a deeper understanding of how electric currents pass through the unit and transfer energy to the process.

Different methods can be applied to produce a bimetallic composite roll. In "Effect of Conductive Circuits on Bonding Quality of Bimetallic Composite Roll Produced by Electroslag Remelting Cladding," Y. Cao et al. investigated the production of a bimetallic composite roll through the electroslag remelting method, in particular evaluating the most efficient circuit configuration through combined modeling and experiment. The different circuit configuration was demonstrated to affect the uniformity of key physical fields in the process such as electrical potential, current density, temperature, Lorentz force, and velocity distribution. The study concluded that the so-called ESTM circuit is the most efficient, and this was demonstrated through a production of bimetallic rolls of $\mathrm{C} 45$ and GCr15 bearing steels.

The design of gas injection lance nozzles for basic oxygen furnace steelmaking and other top-blown converter processes is a complex and intricate art. The understanding of nozzle behavior can be greatly augmented with computational modeling approaches, which generate detailed information about the compressible fluid flow fields in and 
around the nozzle and how they interact with the rest of the furnace. In "Influence of Preheating Temperature on the Characteristics of $\mathrm{O}_{2}+\mathrm{CO}_{2}$ Jet by Mixed Injection with a Swirling Oxygen Nozzle," C. Feng et al. study the process impact of preheating the gas mix fed to a swirling lance nozzle design, drawing a number of conclusions with practical implications for the operation of such systems.
All titles and authors of the articles published under the topic "Computational Modeling in Pyrometallurgy" in the October 2021 issue (vol. 73, no. 10) of JOM can be accessed via the journal's page at: http:// link.springer.com/journal/11837/73/10/page/1.

Publisher's Note Springer Nature remains neutral with regard to jurisdictional claims in published maps and institutional affiliations. 